\title{
Estudo fatorial dos componentes da leitura: velocidade, compreensão e reconhecimento de palavras
}

\author{
Alessandra Gctuzo Seabra - UnivesidadePresditeiana Madkezie, SãoPaulo, São Paulo, Brasil \\ Natália Martins Dias- UnivesidadePresditeiana Madkenzie, SãoPaulo, SãoPaulo, Brasil \\ JośMaria Montid - Anhanguea Eduracional, SãoPaulo, SãoPaulo, Brasil
}

\begin{abstract}
Resumo
Modelos de processamento de informação têm identificado diferentes processos envolvidos na leitura, incluindo reconhecimento de palavras, compreensão e velocidade, podendo o reconhecimento ocorrer por três diferentes estratégias, logográfica, alfabética e ortográfica. De modo a identificar tais componentes na leitura, foram avaliados 443 estudantes da $1^{\mathfrak{a} \text { à }} 4^{\mathrm{a}}$ - série do ensino fundamental, em testes de reconhecimento de palavras, compreensão oral/ auditiva, compreensão de leitura, processamento ortográfico e velocidade de leitura. Foi conduzida análise fatorial por componentes principais e rotação oblimin, derivando quatro fatores. 0 fator 1 incluiu reconhecimento de palavras familiares e compreensão linguística oral e escrita; o fator 2 compreendeu as estratégias logográfica e alfabética de reconhecimento de palavras; o fator 3, a estratégia ortográfica; e o fator 4 referiu-se à velocidade leitora. Os achados, bastante próximos ao esperado teoricamente, podem auxiliar na compreensão dos processos cognitivos envolvidos na leitura de crianças no início do ensino fundamental.
\end{abstract}

Palaurasdhave Leitura; Análise fatorial; Processos cognitivos.

\section{Factorial study of reading components: speed, comprehension and word recognition}

\begin{abstract}
Information Processing Models has identified different process involved in the reading, including word recognition, comprehension and speed, being that the word recognition can occur for three different strategies, the logographic, the alphabetic and ortographic one. In the way to identify such components in the reading, 443 students of the 1st to the 4th grades of the elementary school were evaluated in words recognition, oral/auditory comprehension, reading comprehension, orthographic processing and speed of reading tests. A Factorial Analyses of principal components and oblimin rotation was conducted, deriving four factors. The factor 1 included recognition of familiar words and oral and written linguistic comprehension; the factor 2 understood the logographic and alphabetic strategies of word recognition; the factor 3, the orthographic strategy; and the factor 4 referred to the speed of reading. The finds, near to the expected theoretically, are able to help in the comprehension of the cognitive process involved in the reading of children in the beginning of the elementary education.
\end{abstract}

Keguards Reading; Factorial analysis; Cognitive processes.

\section{Estudio factorial de los componentes de la lectura: velocidad, comprensión y reconocimiento de palabras}

\begin{abstract}
Resumen
Modelos de procesamiento de información han identificado diferentes procesos abarcados en la lectura, incluyendo reconocimiento de palabras, comprensión y velocidad, siendo que el reconocimiento puede ocurrir por tres diferentes estrategias, logográfica, alfabética y ortográfica. Con el fin de identificar tales componentes en la lectura, fueron evaluados 443 estudiantes del primer al cuarto año de la enseñanza básica, en testes de reconocimiento de palabras, comprensión oral/ auditiva, comprensión de lectura, procesamiento ortográfico y velocidad de lectura. Fue conducida análisis factorial por componentes principales y rotación oblimin, derivando cuatro factores. El factor 1 incluyó reconocimiento de palabras familiares y comprensión lingǘstica oral y escrita; el factor 2 comprendió las estrategias logográfica y alfabética de reconocimiento de palabras; el factor 3, la estrategia ortográfica; y el factor 4 se refirió a la velocidad lectora. Los hallazgos, muy próximos al esperado teóricamente, pueden auxiliar en la comprensión de los procesos cognitivos involucrados en la lectura de niños en el inicio de la enseñanza básica.

Palabrasdave Lectura; Análisis factorial; Procesos cognitivos.
\end{abstract}

0 desempenho em leitura tem sido conceituado como produto de duas habilidades, o reconhecimento de palavras e a compreensão, especialmente em estudos a partir da década de 1980 (e.g., Gough \& Tunmer, 1986; Hoover \& Gough, 1990). Tais resultados têm sido corroborados por estudos empregando análise fatorial, como os de Levy e Carr (1990) e de Carroll (1993), segundo os quais o reconhecimento de palavras pode ser definido fatorialmente como independente de outras habilidades linguísticas, como a compreensão.
Assim, esse modelo de processamento cognitivo da leitura postula que a competência leitora é 0 resultado de dois componentes principais, um deles referindo processos específicos à linguagem escrita, enquanto o outro pode ser entendido como uma habilidade mais global. 0 primeiro desses aspectos é 0 reconhecimento de palavras (Cunha, Oliveira \& Capellini, 2010; Gough \& Tunmer, 1986; Navas, Pinto \& Dellisa, 2009; Rotta \& Pedroso, 2006). Embora muitos estudos refiram-se a esse componente como sinônimo de decodificação, esta é apenas uma das estratégias possíveis para tal reconhecimento. 
De fato, o modelo proposto por Frith (1985, 1997) descreve três estratégias que podem ser usadas para obter reconhecimento de palavras bem-sucedido. Segundo a autora, uma primeira estratégia possível para 0 reconhecimento de palavras é a logográfica. Nela a criança trata a palavra como um desenho, procedendo a uma leitura por reconhecimento visual global. Essa estratégia prevalece antes da alfabetização formal e nela a leitura é caracterizada pelo uso de pistas contextuais. A segunda estratégia, a alfabética, implica conhecimento das correspondências entre letras e fonemas e utiliza-se dos processos decodificação (na leitura) e codificação (na escrita). Com recurso a essa estratégia, o leitor é capaz de converter o som em escrita e, assim, é capaz de ler e escrever palavras novas e pseudopalavras. Por fim, na estratégia ortográfica, em virtude de suas experiências de leitura, o leitor constitui um léxico mental ortográfico, tornando-se capaz de ler palavras conhecidas ou familiares por meio de reconhecimento direto, sem necessidade de recorrer à conversão fonológica. 0 desenvolvimento dessa estratégia torna possível o reconhecimento de itens que seriam incorretamente lidos pela aplicação dos processos de decodificação, como as palavras irregulares do ponto de vista grafofonêmico (Frith, 1985, 1997).

Investigações acerca do desenvolvimento dessas estratégias de leitura têm sido conduzidas. Em um desses estudos, Salles e Parente (2002) encontraram que a leitura via rota fonológica é predominante na $2^{\mathrm{a}}$ série do ensino fundamental, enquanto na $3^{\text {a }}$ série, ambas as rotas, fonológica e lexical, estão funcionais e desenvolvidas. Os resultados de Capovilla e Dias (2007) foram similares e as autoras verificaram que, na 1ㅡㅗ série, a leitura ocorreu basicamente por meio da estratégia logográfica, sendo a estratégia alfabética ainda muito incipiente, enquanto que, na $2^{\underline{a}}$ série, a estratégia alfabética apresentou-se mais desenvolvida e, ao mesmo tempo, a estratégia ortográfica começou a se desenvolver. $\mathrm{Na} 3^{\text {a }}$ série, a leitura ocorreu ainda predominantemente pela estratégia alfabética, e a ortográfica apresentou-se mais desenvolvida em relação à série anterior. Apenas na $4^{\underline{a}}$ série 0 padrão de desempenho dos estudantes revelou estratégias alfabética e ortográfica bem estabelecidas quando comparadas às séries anteriores.

Estudos têm corroborado a importância do reconhecimento de palavras para a competência em leitura (Corso \& Salles, 2009; Perfetti, 2007; Salles \& Parente, 2002). Por exemplo, Corso e Salles (2009) observaram, dentre crianças da $2^{2}$ série do ensino fundamental, que aquelas com bom desempenho em compreensão de texto apresentavam também boa pefomance em tarefa de reconhecimento de palavras.
Verificaram também que crianças com pobre desempenho no reconhecimento de palavras não apresentavam bom desempenho em tarefa de compreensão de texto. Os autores concluem que 0 reconhecimento de palavras é necessário para se atingir leitura competente, embora não seja condição suficiente para tanto. Ou seja, para além do reconhecimento de palavras, a leitura competente requer um segundo componente, a compreensão (Gough \& Tunmer, 1986; Navas \& cols., 2009; Rotta \& Pedroso, 2006). Esta se refere à compreensão linguística de forma ampla, incluindo não especificamente a compreensão de leitura, mas também a compreensão auditiva ou oral (Gough \& Tunmer, 1986; Hoover \& Gough, 1990; Navas \& cols., 2009; Rotta \& Pedroso, 2006).

De fato, é plausível supor que a competência de leitura, com a extração de informação de um texto lido, só pode ser atingida se houver tanto o reconhecimento de palavras, o que pode ocorrer por qualquer uma das três estratégias de leitura descritas previamente, quanto 0 acesso ao significado de tais palavras, individualmente e no contexto específico. Alguns estudos têm corroborado de forma consistente a existência dos dois componentes, reconhecimento de palavras e compreensão linguística, no processamento da informação escrita (Aaron, Joshi, Gooden \& Bentum, 2008; Oakhill, Cain \& Bryant, 2003). O estudo de 0 akhill e colaboradores (2003), por exemplo, utilizando-se da técnica de regressão linear múltipla, proveu evidências de que diferentes habilidades cognitivas compõem ou explicam a variância nos dois distintos componentes.

Outros estudos têm corroborado a existência dos fatores reconhecimento de palavras e compreensão linguística. Porém, ao mesmo tempo, têm sugerido que outros fatores também podem ajudar a explicar a competência de leitura (Aaron, Joshi \& Williams, 1999; Hoover \& Gough, 1990; Joshi \& Aaron, 2000). A pesquisa de Hoover e Gough (1990), por exemplo, revelou que somente as habilidades de decodificação e compreensão não explicavam todas as variâncias observadas nos desempenhos de leitura. Em seu estudo, encontraram que a decodificação e compreensão explicavam $73 \%$ da variância do desempenho em leitura na 1aㅗ série e $75 \%$ de variância na $2^{\text {a }}$ série. Suas conclusões apontam para a possibilidade de que outros fatores contribuem para 0 processo de leitura, como aqueles relacionados às habilidades ortográficas e à velocidade de leitura.

Os achados de Aaron e colaboradores (1999) corroboram esse entendimento. Para os autores, embora as habilidades de reconhecimento e compreensão sejam dois componentes nos processos 
de leitura, elas não esgotam as variâncias observadas no desempenho em leitura. Em seu estudo, com estudantes de 3aa $4 \underline{\underline{a}}$ e $6^{\underline{a}}$ séries, análise fatorial evidenciou dois fatores subjacentes ao desempenho em leitura, reconhecimento e compreensão. Porém, quando essas análises foram reconduzidas separadamente para cada série, a estrutura para a $6^{\underline{a}}$ série foi alterada, emergindo um terceiro fator que agrupou as habilidades de processamento ortográfico e velocidade de processamento. Os autores concluíram que duas habilidades principais subjazem ao processo de leitura, reconhecimento e compreensão, porém com processamento ortográfico e velocidade de processamento compondo um terceiro fator, especialmente importante em crianças mais velhas.

Em pesquisa posterior, Joshi e Aaron (2000) verificaram que os componentes reconhecimento de palavras e compreensão podiam explicar 48\% da variância em competência de leitura. Um terceiro fator, velocidade de processamento, aumentou em 10\% a variância explicada. Essa pesquisa foi conduzida com estudantes da $3^{\text {a }}$ série, mostrando que, mesmo em leitores relativamente jovens, a velocidade pode ter um papel importante na competência leitora.

Em estudo mais recente, Aaron e colaboradores (2008) descreveram um modelo mais amplo para explicar a competência em leitura, que inclusive aborda componentes de outras áreas além da cognitiva. Em seu Modelo de Componentes da Leitura (Component Mood of Reading, os autores propuseram que a leitura competente seria resultado da atuação e integridade de três domínios. $\mathrm{O}$ primeiro deles é denominado domínio cognitivo e abarca os componentes reconhecimento de palavras e compreensão; o segundo é o componente psicológico e inclui aspectos motivacionais e emocionais relacionados à aprendizagem, assim como estilo de aprendizagem e lócus de controle; 0 terceiro e último componente é 0 ecológico, que se refere ao ambiente familiar, cultural, escolar e estilo parental que envolvem a criança em processo de aprendizagem da linguagem escrita. Esse modelo é baseado no entendimento dos processos envolvidos na leitura proposto originalmente por Gough e Tunmer (1986), no entanto representa uma ampliação deste último, reconhecendo a importância de outros fatores que podem exercer influência sobre a aprendizagem da linguagem escrita.

Ainda assim, em termos de habilidades cognitivas, tem sido corroborada a existência dos dois componentes para a leitura, reconhecimento de palavras e compreensão linguística. 0 próprio estudo de Aaron e colaboradores (2008), com crianças da $2^{\mathrm{a}}$ à 5a árie, usando como base teórica 0 Modelo de Componentes da Leitura, proveu suporte à existência desses dois componentes, evidenciando que reconhecimento de palavras e compreensão linguística são habilidades independentes no domínio cognitivo de leitura. Nesse estudo, também a velocidade de processamento surgiu como um terceiro componente da competência de leitura, cuja influência, apesar de pequena, não é negligenciável. No entanto, a importância relativa dessa habilidade para a leitura competente tendeu a diminuir na progressão da $2^{\mathrm{a}}$ à $5^{\mathrm{a}}$ série. Para os autores, com a progressão escolar as crianças se tornam mais proficientes no reconhecimento de palavras, o que torna mais difícil isolar esse componente dos efeitos da velocidade ou fluência.

Assim, a habilidade de fluência pode ter um papel mais importante para a leitura competente em crianças nas etapas iniciais da alfabetização e é pertinente que investigações considerem esse componente, uma vez que dificuldades de fluência podem constituir uma das principais características dos maus leitores, levando a uma leitura lenta e, na maioria das vezes, muito trabalhosa (Wolf \& Katzir-Cohen, 2001). A pesar disso, Navas e colaboradores (2009) destacam que apenas recentemente a fluência tem sido foco de preocupação nos contextos clínico e escolar.

Dessa forma, 0 papel da velocidade de processamento não figura muito claro, de modo que algumas pesquisas sugerem que sua importância relativa seja maior em estudantes mais velhos, a partir da 6a série (Aaron \& cols., 1999), enquanto outras apontam para sua influência na competência de leitura de estudantes já na $3^{\underline{a}}$ série (Joshi \& Aaron, 2000). Novos estudos devem, portanto, investigar qual 0 papel da velocidade em um modelo cognitivo da leitura, verificando se essa habilidade compõe um fator isolado deste desempenho em estudantes de distintas séries escolares.

Por sua vez, há também evidências de que esses dois novos componentes, velocidade e processamento ortográfico, estejam relacionados. Por exemplo, Salles e Parente (2002) verificaram que as crianças com melhores habilidades de leitura de palavras foram aquelas que leram 0 texto com maior velocidade. A partir disso as autoras concluíram que, nessas crianças, a leitura de palavras estaria automatizada e, portanto, 0 acesso preciso e rápido ao léxico mental tenderia a influenciar no tempo despendido na leitura. Por sua vez, esse tipo de leitura, com recurso ao léxico mental, é típico do processamento ou estratégia ortográfica, denotando a relação entre esse processamento e a velocidade de leitura de textos.

A relevância da investigação dos modelos de processamento e das habilidades que compõem a competência de leitura repousa na necessidade de 
ampliar a compreensão acerca desses processos e suas inter-relações. Por exemplo, com base em seu estudo, Aaron e colaboradores (1999) sugerem que é possível haver formas de problemas de leitura que resultem de deficiências em processos ortográficos, velocidade de leitura, ou uma combinação dos dois fatores, para além daquelas às quais subjazem déficits no reconhecimento de palavras ou compreensão, em geral mais documentadas na literatura. Em outro estudo do mesmo grupo de pesquisa (Aaron \& cols., 2008), os autores confirmaram que o diagnóstico e a subsequente intervenção baseada no Modelo dos Componentes da Leitura proveu resultados mais satisfatórios do que a baseada no Modelo Tradicional de Discrepância Inteligência-Leitura. Isso porque 0 modelo de componentes permite a identificação dos processos específicos que estão prejudicados e, então, uma intervenção específica para desenvolvimento desses componentes pode ser levada a cabo, com resultados mais satisfatórios.

Conhecer os componentes do processo de leitura pode ter implicações clínicas, na medida em que pode contribuir à compreensão das dificuldades apresentadas por determinadas crianças e à maneira mais adequada de intervenção, preocupação já mostrada por alguns pesquisadores no contexto nacional (e.g. Cunha \& cols., 2010; Navas \& cols., 2009). Pode-se derivar desse conhecimento também implicações educacionais, pois um sistema efetivo de ensino deverá contemplar meios visando estimular e desenvolver todos os componentes e processos da competência em leitura. Perante essa revisão, 0 presente estudo avaliou as distintas habilidades de reconhecimento de palavras, incluindo as três estratégias de leitura propostas por Frith (1985, 1997): logográfica, alfabética e ortográfica. Foram avaliadas também as habilidades de compreensão oral/ auditiva e de leitura e a velocidade de leitura. Buscou-se, assim, investigar os processos envolvidos na competência de leitura em uma amostra de estudantes brasileiros na fase inicial da alfabetização, da $11^{\mathrm{a}}$ à $4^{\underline{a}}$ série do ensino fundamental.

\section{Partiajpantes}

\section{Método}

Participaram deste estudo 443 crianças de ambos os sexos, da 1a à $4^{\mathrm{a}}$ série do ensino fundamental, com idade média de 8,4 anos (DP=1,47). D estas, 104 eram da $1^{\text {a }}$ série $(\mathrm{M}=6,7$ anos; $\mathrm{DP}=0,6), 147$ da $2^{\mathrm{a}}$ série $(\mathrm{M}=8,2 ; \mathrm{DP}=1,1), 83$ da 3a $(\mathrm{M}=8,8 ; \mathrm{DP}=0,8)$ e 109 da $4^{a}$ série $(M=9,9 ; D P=0,9)$. Todas estavam matriculadas em uma escola pública do interior do Estado de São Paulo. Participaram todos os estudantes daquela escola cujos pais o autorizaram, não sendo adotado a prioi qualquer critério de exclusão, tal como histórico de repetência escolar. Não havia na amostra estudantes com deficiência mental ou sensorial conhecida nãocorrigida.

\section{Instrumentos}

Tetede Competênia de Leitura dePalavras ePsandqpalavas - TCLPP

O TCLPP (Seabra \& Capovilla, 2010) avalia a competência de leitura silenciosa. Possui 70 itens de teste, cada qual com um par composto de uma figura e de um item escrito. Os pares figura-escrita de palavras corretas regulares e irregulares devem ser aceitos e aqueles com incorreção semântica ou pseudopalavras devem ser rejeitados. Por exemplo, itens do tipo palavras corretas regulares (CR, ex: palavra FADA, sobre a figura de uma fada), vizinhas semânticas (VS, ex: palavra RÁDIO, sobre a figura de um telefone) e pseudopalavras estranhas (PE, ex: palavra MELOCE, sobre a figura de um palhaço) podem ser lidas corretamente por qualquer uma dentre as três estratégias de leitura. Já as vizinhas visuais (VV, ex: palavra TEIEUISÃO, sobre a figura de uma televisão) e as vizinhas fonológicas (VF, ex: palavra MACHICO, sobre a figura de mágico) não podem ser lidas pela estratégia logográfica, mas somente pelas alfabética e ortográfica, visto que, apesar delas possuírem uma forma visual parecida com a palavra correta, elas devem ser rejeitadas. Logo, erros nesses itens sugerem ausência de leitura pelas estratégias alfabética e ortográfica.

As palavras corretas irregulares (CI, ex: palavra BRUXA, sobre a figura de uma bruxa) podem ser lidas pela estratégia logográfica ou ortográfica, mas não pela alfabética, pois nesse caso a aplicação das regras de correspondência grafofonêmicas levaria a erros por regularização, tendo as palavras a ser rejeitadas. Finalmente, as pseudopalavras homófonas ( $\mathrm{PH}$, ex: palavra TÁCSI, sobre a figura de um táxi) somente podem ser lidas corretamente pela estratégia ortográfica, pois, se aplicadas outras estratégias de leitura, tenderiam a ser aceitas, pois tanto a forma visual quanto auditiva resultante é semelhante à da palavra correta. Assim, erros em PH sugerem ausência de estratégia ortográfica. Dessa forma, o padrão de resposta ao teste pode auxiliar na identificação da natureza particular da dificuldade de leitura de uma determinada criança. Evidências de validade e dados de normatização do instrumento estão publicados em Seabra e Capovilla (2010).

Teste Contrastivo de Compreensão Auditiva e de Leitura TCCAL

O TCCAL (Capovilla \& cols., 2005) avalia as habilidades de compreensão auditiva e de compreensão 
de leitura silenciosa. A comparação entre ambas as habilidades permite realizar o diagnóstico diferencial do distúrbio de aquisição de leitura, diferenciando-o do distúrbio geral de linguagem. 0 instrumento é composto de dois subtestes: Compreensão de Leitura (CL) e Compreensão Auditiva (CA), cada qual com 40 itens de teste, arranjados em ordem crescente de dificuldade. Em cada item, a criança deve escolher, dentre cinco figuras alternativas, aquela que corresponde à sentença ouvida, no caso do subteste CA, ou lida, no caso do CL. Evidências de validade em uma amostra de crianças de $1^{\text {a }}$ série do ensino fundamental estão publicadas em Capovilla e Capovilla (2004) e dados normativos estão disponíveis em Capovilla e colaboradores (2005).

\section{TetedeProcssamento Ortogáfico Computadonizadb- TPOC}

Para avaliação do processamento ortográfico foi usado o TPOC (Montiel \& Capovilla, 2008a), que avalia as habilidades de reconhecimento ortográfico que não dependem de estratégias de conversão grafema-fonema. $\mathrm{O}$ instrumento é computadorizado e possui 15 itens de teste. 0 teste apresenta a instrução inicial "Você ouvirá algumas palavras. Escolha a palavra escrita corretamente". Cada item é constituído por uma palavra emitida pelo programa (digitalizada) e três alternativas de resposta, das quais somente uma é a alternativa correta. As duas outras alternativas são palavras escritas incorretamente, mas que mantêm a mesma forma fonológica da palavra escrita corretamente. A tarefa do sujeito consiste em selecionar com o mouse dentre as opções, a palavra escrita de forma correta. Por exemplo, diante da palavra ouvida faça, as opções apresentadas por escrito serão faça, fasa e fassa Dados sobre a construção do instrumento, incluindo seleção das palavras que 0 compõem, estão publicados em Montiel (2008). Em outro estudo, 0 instrumento apresentou evidências de validade e alta consistência interna (Dias, Montiel \& Seabra, submetido).

\section{TetedeV docidadedeLeitura Computadanzadb- TV LC}

Para avaliar a velocidade de leitura foi usado 0 TVLC (Montiel \& Capovilla, 2008b). 0 teste requer que o sujeito leia o mais rápido que puder as palavras apresentadas na parte central da tela do computador. Os escores do teste fornecem os acertos e erros realizados e o tempo de reação e locução do avaliado. Para esse estudo, foram utilizadas apenas as medidas de tempo de reação (TR) e tempo de locução (TLoc) das respostas dos participantes.

O TVLC consta de 60 itens divididos em quatro partes (de P1 a P4), sendo 15 palavras irregulares (P1), 15 pseudopalavras (P2), 15 palavras relacionadas a conteúdo (i.e., substantivos - P3) e 15 palavras relacionadas à função (i.e., três conjunções, três adjetivos, seis pronomes, dois advérbios e um numeral - P4), apresentadas nessa ordem. Todas as palavras possuem entre três e quatro letras, de modo a manter um padrão relacionado ao tamanho da palavra. Como um estudo exploratório, para o presente artigo não foram utilizados o TR e TLoc individuais de cada parte do TVLC, sendo opção dos autores utilizar uma medida total de TR e TLoc no instrumento.

Dados sobre a construção do instrumento, incluindo a seleção das palavras que o compõem, estão publicados em Montiel (2008). As palavras são apresentadas em letra modelo bastão, de fôrma e maiúsculas, fonte Tims NewRoman, tamanho 72, em tinta preta, e cada uma fica exposta por tempo indeterminado na tela. 0 instrumento é computadorizado e o satware operado pelo aplicador. Maiores informações sobre o TVLC constam na tese original. Em outra pesquisa, apresentaram-se evidências de validade e dados de fidedignidade do instrumento (Dias \& cols., submetido).

\section{Procedimento}

O estudo recebeu aprovação do Comitê de Ética em Pesquisa. Após assinatura do Termo de Consentimento Livre e Esclarecido pelos responsáveis das crianças participantes iniciaram-se as avaliações. Cada instrumento foi aplicado em uma sessão e não sendo mais que um, em cada criança no mesmo dia, de modo a evitar fadiga. 0 Teste de Competência de Leitura de Palavras e Pseudopalavras e o Teste Contrastivo de Compreensão Auditiva e de Leitura foram aplicados coletivamente, na própria sala de aula, em três sessões de duração aproximada de 30 minutos, e os subtestes deste último foram aplicados em duas sessões com intervalo de uma semana entre elas. 0 Teste de Processamento O rtográfico Computadorizado e o Teste de Velocidade de Leitura Computadorizado foram aplicados individualmente em sala disponibilizada pela escola e com recurso a um notbook em uma sessão de aproximadamente 15 minutos.

\section{Resultados}

De modo a investigar os distintos componentes das habilidades de leitura, foi conduzida análise fatorial exploratória por componentes principais e rotação ddimim dada a correlação tanto empírica quanto teórica entre as variáveis em análise. Foram incluídas as seguintes medidas: escore nos subtestes do Teste de Competência de Leitura de Palavras e Pseudopalavras (CR, CI, PE, VS, VV, VF e PH), escore nos subtestes de Compreensão Auditiva e de Leitura do Teste 
Contrastivo de Compreensão Auditiva e de Leitura (CL e CA), escore (TPOC Tot) e tempo de reação (TPOC TR) no Teste de Processamento Ortográfico Computadorizado, tempo de reação (TVLC TR) e tempo locucional (TVLC TL) totais no Teste de Velocidade de Leitura Computadorizado. Nesses parâmetros, a aplicabilidade da análise fatorial foi julgada adequada, com coeficiente $\mathrm{KMO}=0,84$ e teste de esfericidade de Bartlett=0,000. Adotou-se como critério para seleção dos fatores égenvalues maiores ou iguais a 1. 0 gráfico screplté apresentado na Figura 1.

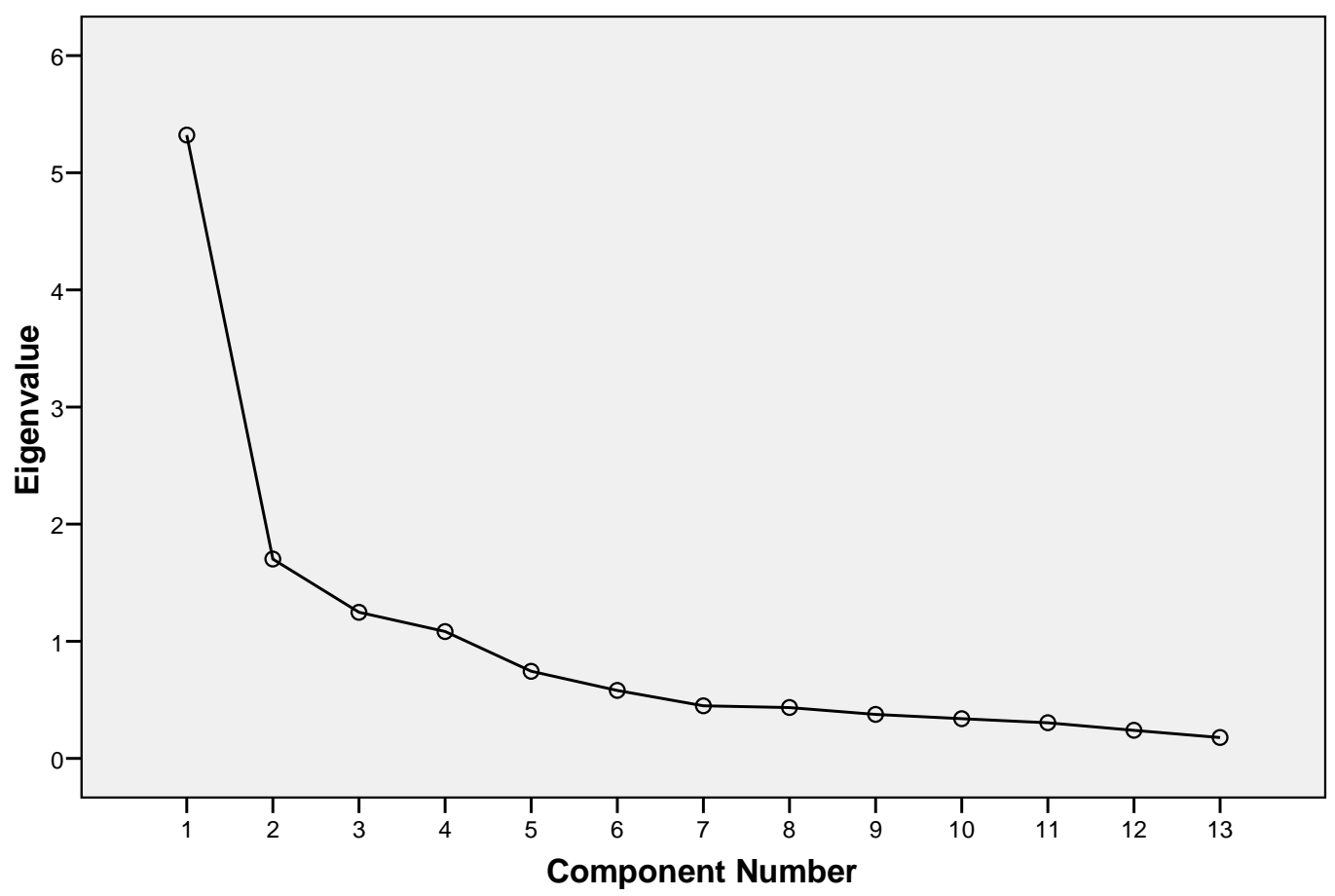

Figura 1. Gráfico Scree plot

Tabela 1. Matriz de cargas fatoriais dos desempenhos nos diversos testes de leitura

\begin{tabular}{lrrrr}
\hline \multirow{2}{*}{ Testes de leitura } & \multicolumn{4}{c}{ Fatores } \\
\cline { 2 - 5 } TCLPP_CI & $\mathbf{0 , 9 1 4}$ & $-0,097$ & $-0,046$ & \multicolumn{1}{c}{4} \\
\hline TCLPP_CR & $\mathbf{0 , 8 7 0}$ & $-0,031$ & $-0,132$ & $-0,150$ \\
TCCAL_CL & $\mathbf{0 , 7 4 4}$ & 0,147 & 0,226 & 0,051 \\
TCCAL_CA & $\mathbf{0 , 5 7 7}$ & $\mathbf{0 , 4 1 7}$ & 0,102 & 0,069 \\
TCLPP_VS & $-0,068$ & $\mathbf{0 , 8 9 7}$ & $-0,062$ & $-0,053$ \\
TCLPP_PE & $-0,044$ & $\mathbf{0 , 8 5 8}$ & 0,082 & $-0,095$ \\
TCLPP_VV & $\mathbf{0 , 3 6 8}$ & $\mathbf{0 , 5 9 1}$ & 0,118 & $-0,014$ \\
TCLPP_PH & $-0,197$ & 0,047 & $\mathbf{0 , 9 4 6}$ & 0,079 \\
TCLPP_VF & 0,157 & 0,239 & $\mathbf{0 , 6 0 3}$ & 0,006 \\
TPOC-Tot & $\mathbf{0 , 4 2 1}$ & $-0,068$ & $\mathbf{0 , 5 4 1}$ & $-0,101$ \\
TPOC_TR & $-0,120$ & 0,115 & $\mathbf{- 0 , 5 1 1}$ & $\mathbf{0 , 3 2 8}$ \\
TVLC_TL & 0,090 & $-0,197$ & 0,065 & $\mathbf{0 , 8 8 2}$ \\
TVLC_TR & $-0,116$ & 0,065 & $-0,060$ & $\mathbf{0 , 7 8 6}$ \\
\hline Eignvaluk & 5,322 & 1,702 & 1,247 & 1,083 \\
\% Variância explicada & 40,937 & 13,094 & 9,595 & 8,333 \\
\% Variância acumulada & 40,937 & 54,031 & 63,626 & 71,959 \\
\hline
\end{tabular}


A análise retornou uma solução de quatro fatores, os quais explicaram $71,96 \%$ da variância total em competência de leitura. As cargas fatoriais associadas a esses fatores são apresentadas na Tabela 1. Para facilitar a visualização das habilidades que compõem os distintos fatores, as cargas fatoriais acima de 0,30 estão destacadas em negrito. Encontra-se, a seguir, uma análise compreensiva de cada um dos fatores.

$O$ fator 1 agrupou os desempenhos nos itens "corretas irregulares" (CI) e "corretas regulares" (CR) do Teste de Competência de Leitura de Palavras e Pseudopalavras, o que denota uma carga sobre a competência de leitura, quanto a reconhecimento de palavras. Os desempenhos em Compreensão de Leitura e Compreensão Auditiva, também com cargas no fator 1, atribuem ao mesmo uma carga da habilidade linguística de compreensão. Ainda, apesar de apresentar cargas fatoriais menores, também os itens "vizinhas visuais" (VV), do Teste de Competência de Leitura de Palavras e Pseudopalavras e escore no Teste de Processamento Ortográfico Computadorizado, agruparam-se neste fator. D esse modo, em uma análise inicial, este fator figura mais inespecífico, abarcando habilidades de reconhecimento de palavras familiares e de compreensão. Porém, 0 fator incluiu os desempenhos tanto em compreensão oral quanto em compreensão leitora, corroborando a noção de um componente mais global e não restrito aos processos de leitura.

Dessa forma, esse primeiro fator poderia ser compreendido primariamente como um componente de compreensão linguística. Os demais itens agrupados sobre este fator, corretas regulares, irregulares e vizinhas visuais do Teste de Competência de Leitura de Palavras e Pseudopalavras e Teste de Processamento O rtográfico Computadorizado, podem corroborar este entendimento, pois são em maioria palavras familiares (exceto as vizinhas visuais) e, conforme revisão teórica antes vista, 0 reconhecimento de palavras é habilidade necessária para se alcançar a compreensão de um texto. Ainda assim, de forma mais cautelosa, considera-se este um fator de compreensão linguística e reconhecimento de palavras familiares.

0 fator 2 incluiu escores nos itens "vizinhas semânticas" (VS) e "pseudopalavras estranhas" (PE) do Teste de Competência de Leitura de Palavras e Pseudopalavras com cargas relativamente altas, denotando a este fator forte carga de processamento logográfico. Adicionalmente, a inclusão de itens "vizinhas visuais", do Teste de Competência de Leitura de Palavras e Pseudopalavras neste fator sugere que ele compreende também algum processamento pela estratégia alfabética (decodificação), pois este tipo de item não pode ser corretamente lido pela estratégia logográfica, e a ortográfica, que também poderia ser usada na leitura deste item, compôs um fator distinto. Dessa forma, o fator 2 foi compreendido como um componente de reconhecimento de palavras que envolve principalmente 0 processamento logográfico, mas, em carga menor, também 0 alfabético. 0 desempenho no subteste Compreensão Auditiva do Teste Contrastivo de Compreensão Auditiva e de Leitura também teve carga nesse fator, provavelmente porque os itens que envolvem leitura logográfica e alfabética, conforme avaliados pelo TCLPP, também exigem compreensão para serem corretamente respondidos. Apesar disso, tal item apresentou carga fatorial modesta neste fator e carga mais consistente no fator 1.

O fator 3 apresentou carga fatorial alta oriunda do subteste "pseudopalavras homófonas" (PH), do Teste de Competência de Leitura de Palavras e Pseudopalavras. Outras cargas bastante expressivas agrupadas neste fator foram itens "vizinhas fonológicas" (VF) do mesmo teste e escore e TR do Teste de Processamento Ortográfico Computadorizado. A variável TPOC_TR_Tot apresentou carga negativa, por ser uma medida de tempo. D estaca-se que a variável TPOC Tot pontuou neste fator 3 e também, com carga menor, no fator 1, provavelmente porque está relacionada especificamente à leitura ortográfica, mas também é importante para a leitura em geral, como a leitura de frases com compreensão. Tomados em conjunto, estes resultados permitem compreender o fator 3 como saturado da habilidade de processamento ortográfico.

Já 0 fator 4 agrupou os desempenhos, quanto a tempo de reação e tempo de locução, do Teste de Velocidade de Leitura Computadorizado e, de forma mais moderada, tempo de reação no Teste de Processamento Ortográfico Computadorizado. O u seja, este fator é predominantemente um fator de velocidade de leitura.

\section{Discussão}

Como sumariado na introdução deste artigo, estudos apontam uma dissociação entre as habilidades de reconhecimento de palavras e compreensão (Aaron \& cols., 2008; Carrol, 1993; Levy \& Carr, 1990; O akhill \& cols., 2003). E, ainda, outros sugerem novos componentes do desempenho em leitura (Aaron \& cols., 1999; Aaron \& cols., 2008; Joshi \& Aaron, 2000). Os resultados aqui encontrados são parcialmente condizentes com essas pesquisas prévias

Em consonância com estes estudos (Aaron \& cols., 1999; Aaron \& cols., 2008; Carrol, 1993; Joshi \& Aaron, 2000; Levy \& Carr, 1990), dentre os quatro 
fatores encontrados na presente pesquisa, a compreensão (tanto oral quanto de leitura) situou-se em um fator (fator 1), e as habilidades de reconhecimento de palavras relacionadas às estratégias de leitura agruparam-se distintamente em outros fatores, principalmente as estratégias logográfica (fator 2) e ortográfica (fator 3). Por fim, conforme também esperado teoricamente (Aaron \& cols., 1999; Aaron \& cols., 2008), figurou um fator de velocidade de leitura (fator 4).

No que tange estritamente ao reconhecimento de palavras, esses achados recebem sustentação teórica do modelo de Frith $(1985,1997)$, uma vez que os fatores 2 e 3, respectivamente, diferenciaram as estratégias logográfica e ortográfica, descritas pela autora. Este achado por si só representa um ganho em relação às pesquisas prévias que, em geral, têm considerado 0 componente reconhecimento de palavras de forma inespecífica ou unitária, sem considerar as distintas estratégias de leitura que podem ser utilizadas para esse reconhecimento.

Especificamente com relação ao fator 1, compreensão linguística, os resultados oferecem sustentação à compreensão desse componente como uma habilidade linguística mais ampla, uma vez que agrupou tanto o desempenho em compreensão de leitura como também compreensão oral (Gough \& Tunmer, 1986; Hoover \& Gough, 1990; Rotta \& Pedroso, 2006). Por outro lado, o fator também deteve cargas de desempenhos relacionados ao reconhecimento de palavras, sobretudo palavras familiares (corretas, tanto regulares quanto irregulares, no Teste de Competência de Leitura de Palavras e Pseudopalavras e Teste de Processamento Ortográfico Computadorizado). É provável que essa configuração tenha se dado devido à relação entre esses itens e a habilidade de compreensão de leitura que integrou 0 mesmo fator, pois, por serem palavras propriamente (e não pseudopalavras), esses itens podem ser processados semanticamente. Esta solução reforça a relação entre habilidades de reconhecimento de palavras e a compreensão de leitura, sustentada nos estudos de Corso e Salles (2009), Perfetti (2007) e Salles e Parente (2002), oferecendo respaldo à conclusão daqueles autores de que o reconhecimento é de fato habilidade necessária à compreensão de leitura.

Sobre os outros componentes que integram 0 desempenho em leitura, tais como os sugeridos por Aaron e colaboradores (1999), este estudo também traz alguns achados. De fato, já na década de 1990, estudos como os de Hoover e Gough concluíam que somente o reconhecimento de palavras (por decodificação) e a compreensão não explicavam todas as variâncias observadas no desempenho de leitura, tendo outras habilidades contribuído neste processo, como as de processamento ortográfico e a velocidade de leitura. Os achados deste estudo corroboram esses autores. Os componentes evidenciados na solução fatorial contemplam, além de fatores de compreensão e reconhecimento de palavras (logográfico e alfabético), componentes de processamento ortográfico e velocidade de leitura.

Conforme foi evidenciado, os quatro fatores extraídos neste estudo possuem poder explicativo de $71,96 \%$ da variância total em competência de leitura. Somente os dois primeiros fatores já detêm $54,03 \%$ da variância explicada do modelo, corroborando a noção de que os componentes de compreensão e reconhecimento de palavras seriam os principais elementos em um modelo de processamento da leitura (Aaron \& cols., 1999; Aaron \& cols., 2008; Gough \& Tunmer, 1986; Hoover \& Gough, 1990). Porém, o fato de 0 fator 1 não ser um componente puro de compreensão (pois também abarca 0 reconhecimento de palavras familiares) impossibilita delimitar qual a real importância relativa desses dois componentes.

Por sua vez, as habilidades de processamento ortográfico e velocidade, mencionadas por Aaron e colaboradores (1999), Hoover e Gough (1990) e Joshi e Aaron (2000), que constituíram nesta pesquisa os fatores 3 e 4, explicam, respectivamente, 9,56\% e $8,33 \%$ da variância total desse desempenho. A variância explicada por estes componentes é modesta, porém significativa, sugerindo a necessidade de considerar tais componentes em modelos de processamento da leitura de crianças no início da alfabetização. É possível que, em estudantes mais velhos, tais componentes detenham uma menor variância explicada dentro do modelo de processamento, pois, à medida que os alunos tornamse mais proficientes, é provável que essas habilidades não sejam facilmente diferenciadas entre si e componham um único componente de reconhecimento de palavras. Essa suposição, compartilhada pelos resultados de Aaron e colaboradores (2008), deve ser investigada em estudos futuros, porém é coerente teoricamente (Frith, 1985, 1997), pois com a progressão das séries escolares e 0 desenvolvimento da estratégia ortográfica, a leitura de qualquer palavra que lhe seja conhecida passa a ocorrer predominantemente por esta estratégia, o que também confere ao leitor maior velocidade e fluência. Finalizando esta discussão, os resultados da análise fatorial soaram parcialmente consistentes com a literatura e estes achados provêm dados relevantes às teorias e modelos de processamento da informação ao delinear as habilidades envolvidas no processo de leitura. 


\section{Considerações finais}

O estudo explorou os processos envolvidos na leitura de crianças na fase inicial da alfabetização ante 0 referencial de modelos de processamento da informação que postulam a existência de distintos componentes envolvidos na competência leitora. Os resultados foram parcialmente condizentes com 0 esperado, conforme relato da literatura. Foram encontrados quatro fatores, sendo o primeiro um componente de compreensão linguística que incluiu também 0 reconhecimento de palavras familiares. 0 segundo fator compreendeu as estratégias logográfica e, em menor carga, alfabética de reconhecimento de palavras; o terceiro fator abarcou o processamento ortográfico e 0 quarto fator foi referido como velocidade de leitura. Os resultados mostram que distintas habilidades compõem o desempenho em leitura e corroboram a noção de que todas essas habilidades devem ser abarcadas em um processo de avaliação.

Tal como mencionado na introdução, é possível que alguns quadros de problemas de leitura e escrita sejam caracterizados por déficits em habilidades de processamento ortográfico ou velocidade de leitura, com habilidades relativamente preservadas de reconhecimento de palavras ou compreensão, que em geral têm recebido maior atenção dos pesquisadores. Este estudo expande sua contribuição à área ao apresentar instrumentos desenvolvidos com a finalidade de avaliar especificamente tais aspectos. Pesquisas futuras devem ampliar estes achados, avaliando e investigando os componentes da leitura em crianças mais velhas, nas quais a importância relativa das habilidades de velocidade e processamento ortográfico pode ser diferente. Neste âmbito, cabe questionar se os modelos de processamento da leitura deveriam diferenciar-se e abranger distintos níveis desenvolvimentais. Ou seja, é plausível que os processos cognitivos que subjazem à leitura competente divirjam dos processos envolvidos na leitura iniciante, como em estudantes de $1^{\mathrm{a}}$ a $4^{\underline{\mathrm{a}}}$ série, a exemplo deste estudo. Uma hipótese a ser testada é que, em leitores competentes, as habilidades aqui investigadas estejam mais indiferenciadas, com os componentes de velocidade e processamento ortográfico não se diferenciando de outras estratégias.

Dentre as limitações deste estudo cabe citar 0 reduzido número amostral por série escolar, que inviabilizaria a condução de análises separadamente por série, 0 que poderia prover resultados ainda mais interessantes. Por fim, é notório que o conhecimento acerca destes componentes tem importância teórica no que tange a uma melhor compreensão dos processos cognitivos envolvidos na leitura e, sob este aspecto, o presente estudo corrobora a noção de um modelo multidimensional do processamento envolvido na leitura. Por outro lado, sua importância prática repousa na possibilidade de identificação de perfis de maus leitores ou das dificuldades subjacentes a seus desempenhos, de modo a contribuir no direcionamento de intervenções mais adequadas a cada caso. Espera-se que estudos futuros possam avançar nesta direção e, desse modo, possam contribuir à identificação de perfis de maus leitores e à disponibilização de instrumentos para avaliação de cada uma dessas habilidades.

\section{Referências}

Aaron, P. G., Joshi, R. M., Gooden, R., \& Bentum, K. E. (2008). Diagnosis and treatment of reading disabilities based on the Component Model of Reading: an alternative to the Discrepancy Model of LD. Jaumal of LeamingDisabilities 41, 67-84.

Aaron, P. G., Joshi, R. M., \& Williams, K. A. (1999). Not all reading disabilities are alike. Jaumal of LemingDisabilities, 32(2) 120-137.

Capovilla, A. G. S., \& Capovilla, F. C. (2004). Alfabetizaçãa míabofônica São Paulo: Memnon.

Capovilla, A. G. S., \& Dias, N. M. (2007). Desenvolvimento de estratégias de leitura no ensino fundamental e correlação com nota escolar. Psicolia emReista, 13, 63-82.

Capovilla, F. C., Viggiano, K., Capovilla, A. G. S., Raphael, W. D., Bidá, M. C. P. R., Neves, M. V., \& Mauricio, A. C. (2005). Como avaliar o desenvolvimento da compreensão de leitura de sentenças em surdos do ensino fundamental ao médio, e analisar processamento sintático para extração de significado: versão original validada e normatizada do Teste de Competência de Leitura de Sentenças. Em F. C. Capovilla \& W. D. Raphael (Orgs.), Enidopédia da língua de sinais Brasilera: o mundo do surdb em libras (pp. 405-857, vol. 3). São Paulo: Edusp.

Carroll, J. (1993). Human cogitive abilities a surve of factor-analytic studes Nova Iorque: Cambridge University Press.

Corso, H. V., \& Salles, J. S. (2009). Relação entre leitura de palavras isoladas e compreensão de leitura textual em crianças. Letras deHge, 44(3), 2835.

Cunha, V. L. O ., Oliveira, A. M., \& Capellini, S. (2010). Compreensão de leitura: princípios avaliativos e 
interventivos no contexto educacional. Revista Teias, 11(23), 221-240.

Dias, N. M., Montiel, J. M., \& Seabra, A. G. (submetido). Instrumentos de avaliação da leitura e escrita: investigação de seus parâmetros psicométricos. Avanesen Psicdoǵa Latimoamericana.

Frith, U. (1985). Beneath the surface of developmental dyslexia. Em K. Patterson, J. Marshall \& M. Coltheart (O rgs.), Sunfare dyslexia: narropsyddogical and cogitive sudies of phondogical reading (pp. 301330). Londres: L. E. Associates.

Frith, U. (1997). Brain, mind and behavior in dyslexia. Em C. Hulme \& M. Snowling (Eds.), Dydexia: bidogy, cognitionandintervetion (pp. 01-19). Londres, UK: Whurr Publishers.

Gough, P., \& Tunmer, W. (1986). D ecoding, reading and reading disability. Remeelial and Speial Eduration, 7(1), 6-10.

Hoover, W., \& Gough, P. (1990). The simple view of reading. Reading\& Writing 2, 127-160.

Joshi, R. M., \& Aaron, P. G. (2000). The component model of reading: simple view of reading made a little more complex. Reading Psychdogy, 21(2), 8597.

Levy, B. A., \& Carr, T. H. (1990). Component process analysis: conclusions and challenges. Em T. H. Carr \& B. A. Levy (O rgs.), Reading and its dedopment: component skills appoadhes (pp. 280-284). Nova Iorque: Academic Press.

Montiel, J. M. (2008). Evidânias de validade de testes para identificar componentes da lingragem escrita (Tese de D outorado). Programa de Pós-Graduação Strido Sensu em Psicologia, Universidade São Francisco, Itatiba, Brasil.

Montiel, J. M., \& Capovilla, A. G. S. (2008a). Tetede Procssamento Ortogáfico Computadanzado - TPOC. Software desenvolvido.
Montiel, J. M., \& Capovilla, A. G. S. (2008b). Teste de V docidade de Leitura computadbrizadb - TVLC. Software desenvolvido.

Navas, A. L. G. P., Pinto, J. B. C. R., \& Dellisa, P. R. R. (2009). Avanços no conhecimento do processamento da fluência em leitura: da palavra ao texto. Revista da Sociedade Brasilèra de Foncaudidoga, 14(3), 553-559.

Oakhill, J. V., Cain, H., \& Bryant, P. E. (2003). The dissociation of word reading and text comprehension: evidence from component skills. Langageand cognitiveprosses, 18(4), 443-468.

Perfetti, C. (2007). Reading ability: lexical quality to comprehension. Saintific studes of reading 11(4), 357-383.

Rotta, N. T., \& Pedroso, F. S. (2006). Transtorno da linguagem escrita-dislexia. Em N. T. Rotta, L. O hlweiler \& R. S. Riesgo (Orgs.), Trantamos da aprendzagm abordagmnnerrdidógica emiltidsipinar (pp. 151-164). Porto Alegre: Artmed.

Salles, J. F., \& Parente, M. A. M. P. (2002). Processos cognitivos na leitura de palavras em crianças: relações com compreensão e tempo de leitura. Psicolga: ReflexãoeCńtica, 15(2), 321-331.

Seabra, A. G., \& Capovilla, F. C. (2010). Teste de Comptência de Leitura de Palavas e Psendqpalavas São Paulo: Memnon.

Wolf, M., \& Katzir-Cohen, T. (2001). Reading fluency and its intervention. Saientific Studes of Reading 5, 211-239. 
Sobre os autores:

Alessandra Gotuzo Seabra é psicóloga, mestre, doutora e pós-doutorada em Psicologia Experimental pela Universidade de São Paulo, docente do Programa de Pós-Graduação em Distúrbios do Desenvolvimento da Universidade Presbiteriana Mackenzie, São Paulo, coordenadora do Grupo de Neuropsicologia Infantil (www.neuropsiinfantil.wordpress.com) e bolsista de produtividade do CNPq.

Natália Martins Dias é psicóloga pela Universidade São Francisco, mestre e doutoranda do Programa de Pósgraduação em Distúrbios do Desenvolvimento da Universidade Presbiteriana Mackenzie (UPM), São Paulo. Professora convidada do programa de Pós-G raduação Lato Sensuem Psicopedagogia da UPM e bolsista FAPESP.

José Maria Montiel é psicólogo, especialista em Diagnóstico e Triagem, mestre e doutor em Avaliação Psicológica. E membro do Programa de Pós-Graduação e Núcleo de Pesquisa do Instituto de Pesquisas Aplicadas e D esenvolvimento Educacional (IPADE) - Anhanguera Educacional.

Contato com os autores:

Rua da Consolação, 930 - PPG-Distúrbios do D esenvolvimento (prédio 38) - Consolação - CEP 01302-907. São Paulo - SP.

E-mail: alessandragseabra@gmail.com 